\title{
Heart Rate Estimation from Dual Pressure Sensors of a Dialysis Machine
}

\author{
Mattias Holmer ${ }^{1,2}$, Frida Sandberg ${ }^{1}$, Kristian Solem $^{2}$, Bo Olde ${ }^{2}$, Leif Sörnmo ${ }^{1}$ \\ ${ }^{1}$ Department of Biomedical Engineering, Lund University, Sweden \\ ${ }^{2}$ Baxter International Inc., Research Department, Lund, Sweden
}

\begin{abstract}
Dialysis patients often suffer from cardiovascular diseases, motivating the use of continuous monitoring of cardiac activity in clinical routine. Cardiac pressure pulses propagate through the vascular system and enter the extracorporeal blood circuit of a dialysis machine, where the pulses are captured by pressure sensors. The cardiac pulses are obscured by the much stronger pressure pulses originating from the peristaltic blood pump. We have previously shown that a cardiac signal can be extracted from the venous pressure signal. However, that method has been found to perform less well at very low cardiac pressure pulse amplitudes. In the present study, we propose a novel method which addresses this issue by using the signals from both the arterial and the venous pressure sensors. The method is compared to the previous method on clinical data using a photoplethysmogram as reference. The results suggests that heart rate can be estimated more accurately from pressure signals with lower cardiac signal amplitude when both arterial and venous pressure are used, compared to when only the venous signal is used.
\end{abstract}

\section{Introduction}

Severe kidney failure leads to impairment of the vital mechanisms responsible for waste removal and fluid balance in the body. Lost kidney function is often replaced by hemodialysis treatment typically performed thrice a week. Continuous intradialytic determination of physiological information such as heart rate, blood pressure, and respiration rate, can significantly contribute to improved patient monitoring during dialysis. Cardiac monitoring is of special interest since cardiovascular diseases cause almost $50 \%$ of all deaths [1]. Better understanding of cardiac events during dialysis treatment is important for improved patient management as it may lead to modifications of the hemodialysis prescription and reduced disease burden $[2,3]$. Although ECG is sometimes recorded for research purposes, it is not part of clinical routine. As a consequence, basic information on heart rate is not continuously presented to the clinical staff, far less information on

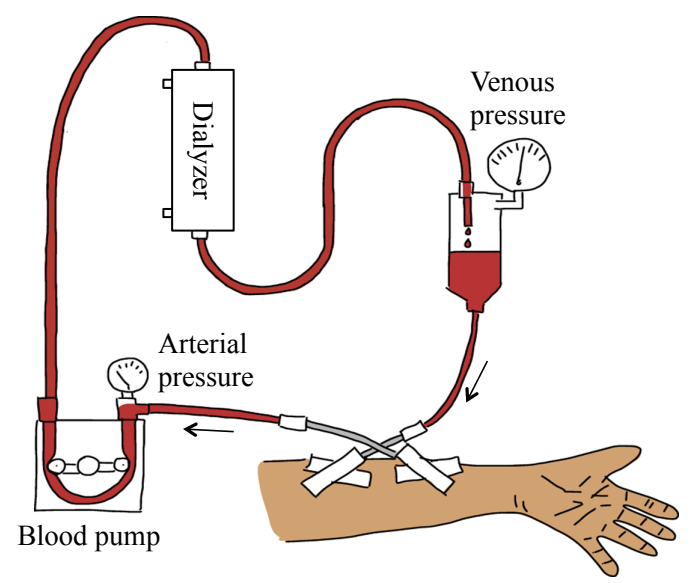

Figure 1. The extracorporeal blood circuit of a dialysis machine.

complex arrhythmias.

It is thus desirable to take advantage of the built-in sensors of the hemodialysis machine to extract cardiac information. Pressure pulses caused by heartbeats propagate in the body and enter the extracorporeal blood circuit of the dialysis machine, where they are measured by the pressure sensors connected to the arterial (where blood leaves the patient) and venous lines (where blood enters the patient), see Fig. 1. A peristaltic blood pump, located between the pressure sensors, generates the desired blood flow. The blood pump is associated with strong periodic pressure pulses, and in the blood circuit their amplitudes are much larger than the amplitudes of the pulses caused by cardiac activity. The pump pressure amplitudes, being around $30 \mathrm{mmHg}$ peak to peak, are up to 100 times larger than the amplitude of the cardiac signal.

In general the problem of extracting cardiac information from the extracorporeal pressure sensors has received little attention in the scientific literature. In two studies, Moissl et al. proposed different approaches to the estimation of heart rate from the arterial pressure sensor [4,5]. Both these studies offered sparse details on methodology, and performance was only evaluated through a few examples.

We have previously shown that the cardiac pressure sig- 
nal can be extracted from the venous pressure signal of a dialysis machine [6]. Here we present an novel method for extracting the common cardiac component present in both the arterial and the venous extracorporeal pressure signals. The proposed method enables estimation of the occurrence times of each heartbeat. The objective of this study is to investigate the performance of heart rate estimation during treatment.

\section{Method}

The main idea behind the proposed method is to iteratively, for $j=0, \ldots, J$, alternate between computing separate pump component estimates $\hat{p}_{a}^{(j)}(t)$ and $\hat{p}_{v}^{(j)}(t)$ for the arterial and venous pressure signals $y_{a}(t)$ and $y_{v}(t)$, respectively, and computing a cardiac component estimate $\hat{c}^{(j)}(t)$ common in the arterial and venous pressure signals. The common cardiac estimate $\hat{c}^{(j)}(t)$ is determined by averaging of an arterial cardiac estimate $\hat{c}_{a}^{(j)}(t)$ and a time shifted venous cardiac estimate $\hat{c}_{v}^{(j)}(t)$. The time shift $\Delta t$ is determined as the delay giving the highest covariance between $\hat{c}_{a}^{(j)}(t)$ and $\hat{c}_{v}^{(j)}(t+\Delta t)$. The cardiac estimates $\hat{c}_{a}^{(j)}(t)$ and $\hat{c}_{v}^{(j)}(t)$ are obtained by subtracting modeled pump signals $\tilde{p}_{a}^{(j)}(t)$ and $\tilde{p}_{v}^{(j)}(t)$ from the respective observed pressure signals $y_{a}(t)$ and $y_{v}(t)$. The pump estimates $\hat{p}_{a}^{(j)}(t)$ and $\hat{p}_{v}^{(j)}(t)$ are determined by subtracting the common cardiac estimate $\tilde{c}^{(j)}(t)$ from $y_{a}(t)$ and $y_{v}(t)$, respectively. The pump estimates are in turn used to create modeled pump signals $\tilde{p}_{a}^{(j)}(t)$ and $\tilde{p}_{v}^{(j)}(t)$. For each iteration, the modeled signals are refined, which gradually decreases the amount of pump signal remainders in the cardiac signal estimate. The iteration continues until the difference in successive estimates is sufficiently small.

The pump profiles $\bar{p}_{x q}^{(j)}(t)$ characterizes the changes in pressure that occur during one roller period (equal to half a revolution of the blood pump) in the arterial ( $a$ ) and venous $(v)$ pressure signals respectively. The subscript $x \in\{a, v\}$ indicates whether the profile belongs to the arterial or the venous pump signal, and the subscript $q \in\{1,2\}$ indicates to which of the two rollers the profile is associated.

The continuous modeled pump signals $\tilde{p}_{a}^{(j)}(t)$ and $\tilde{p}_{v}^{(j)}(t)$ are created by concatenation of pump profiles. Before the concatenation the pump profiles $\bar{p}_{a q}^{(j)}(t)$ and $\bar{p}_{v q}^{(j)}(t)$ are time scaled to fit between the roller period onset times. In a least squares sense, the optimal estimates of the roller period onset times $t_{x, k}^{(j)}, x \in\{a, v\}$ are given by

$$
\hat{t}_{x, k}^{(j)}=\arg \min _{t_{x, k}^{(j)}} \int \frac{\partial^{2} \epsilon_{x}^{2}\left(t ; t_{x, k}^{(j)}\right)}{\partial t^{2}} d t,
$$

where the residual $\epsilon_{x}\left(t ; t_{x, k}^{(j)}\right)$ is defined as

$$
\epsilon_{x}\left(t ; t_{x, k}^{(j)}\right)=\hat{p}_{x}^{(j)}(t)-f\left(\bar{p}_{x 1}^{(j-1)}(t), \bar{p}_{x 2}^{(j-1)}(t) ; t_{x, k}^{(j)}\right),
$$

and the function $f(\cdot)$ gives the pump profile of a complete revolution. The function $f(\cdot)$ is defined by concatenation of $\bar{p}_{x 1}^{(j-1)}(t)$ and $\bar{p}_{x 2}^{(j-1)}(t)$, once these pump profiles have been time-scaled to fit the two intervals $\left[\hat{t}_{x, k-1}^{(j)}, \hat{t}_{x, k}^{(j)}\right]$ and $\left[\hat{t}_{x, k}^{(j)}, \hat{t}_{x, k+1}^{(j-1)}\right]$, respectively. The idea behind minimizing the second derivative of the squared residual is to obtain a smooth cardiac signal estimate.

Note that the optimization is performed sequentially, i.e. for each $k$ :th roller period at a time. Once new roller period onset times are calculated, the pump profiles are updated by normalizing the duration of each pump roller segment prior to calculating the median. The pump model signal $\tilde{p}_{x}^{(j)}(t)$ is then produced by alternating the concatenation of the two pump profiles, i.e., for every other roller period the first pump profile is used,

$$
\tilde{p}_{x}^{(j)}\left(\hat{a}_{x 1, k}^{(j)} t+\hat{t}_{x, 2 k}^{(j)}\right)=\bar{p}_{x 1}^{(j)}(t), \quad 0 \leq t<\bar{T}_{x 1},
$$

and otherwise the second pump profile,

$\tilde{p}_{x}^{(j)}\left(\hat{a}_{x 2, k}^{(j)} t+\hat{t}_{x, 2 k+1}^{(j)}\right)=\bar{p}_{x 2}^{(j)}(t), \quad \bar{T}_{x 1} \leq t<\left(\bar{T}_{x 1}+\bar{T}_{x 2}\right)$.

where the time scale factors $\hat{a}_{x q, k}^{(j)}$ and the average roller period lengths $\bar{T}_{x 1}$ and $\bar{T}_{x 2}$ are determined from the roller period onset times $t_{x, k}^{(j)}$.

To start the iterative process, initial estimates of the pump roller onset times $t_{x, k}^{(0)}$ are needed. These are obtained by finding the time instant of the maximum absolute derivative for each roller period. The initial search interval for the roller periods is estimated by finding the peak in the autocorrelation of the observed signal $y_{x}(t)$.

\section{Validation}

The method was validated on clinical data from 9 patients who underwent regular dialysis treatment by Gambro AK 200 machines. The data was acquired at the Skåne University Hospital, Lund, in a study approved by the local ethical review board. A finger pulse oximeter (LifeSense $(\mathrm{B})$ ) sensor, measuring a photoplethysmographic (PPG) signal, was used as a reference for heart rate. Extracorporeal venous and arterial pressure signals were sampled with external pressure sensors connected to the same sites as the sensors of the dialysis machine. In each patient 20 sections of 1-minute duration with constant blood flow and sufficient signal quality in the PPG reference signal were selected for evaluation. All signals were processed using a sampling rate of $100 \mathrm{~Hz}$. 
The performance of the method using both arterial and venous signals and the previous method using only the venous was evaluated with respect to average heart rate estimation for each 1-min section, with heart rate determined from the PPG-signal as reference. The heart rate calculation was based on heartbeat onset times. The heartbeat onset times was calculated as the time instant of the mid amplitude point of each heartbeat for both the common cardiac component $\hat{c}^{(J)}(t)$ and the PPG-signal [7]. The peak to peak amplitude of each heartbeat was computed as the difference in amplitude of the peak succeeding the mid amplitude point, and the valley preceding the mid amplitude point. Ectopic beats and the succeeding beat was excluded when calculating the average heart rate.

\section{Results}

The method was tested on pressure recordings from dialysis patients. An example of the observed arterial and venous pressure signals $y_{x}(t)$, the final arterial and venous cardiac estimates $\hat{c}_{x}^{(8)}(t)$, the final common cardiac estimate $\hat{c}^{(8)}(t)$, and the reference PPG-signal are displayed in Fig. 2.

Each separate point in Fig. 3 represents the difference between heart rate determined from the PPG signal and the heart rate estimated using both the arterial and venous signals during the 1-min sections.

As a comparison, the difference between the heart rate determined from the PPG signal and the heart rate based on the venous signal only can be seen in Fig. 4. Note the difference in the vertical scales.

In Fig. 5, the absolute deviation from the reference heart rate is plotted versus the estimated average cardiac peak to peak amplitude for each 1-min section. Note the large difference at low cardiac signal amplitudes $(0.2 \mathrm{mmHg})$. It can be shown that the outliers in Fig. 4 corresponds to signals with low cardiac component amplitude.

\section{Conclusions}

A novel iterative method for extraction of the common heart component present in the arterial and venous pressure signals of a dialysis machine has been presented. Initial results suggests that the use of both signals improves detection of heart rate, compared to using only the venous pressure signal. The new method enables continuous online monitoring of a patient's heart rate also for patients with low cardiac signal amplitude.

\section{Acknowledgements}

The authors are grateful to Mårten Segelmark, Lena Mattsson, and Sarok Said at "Filialen", Lund University
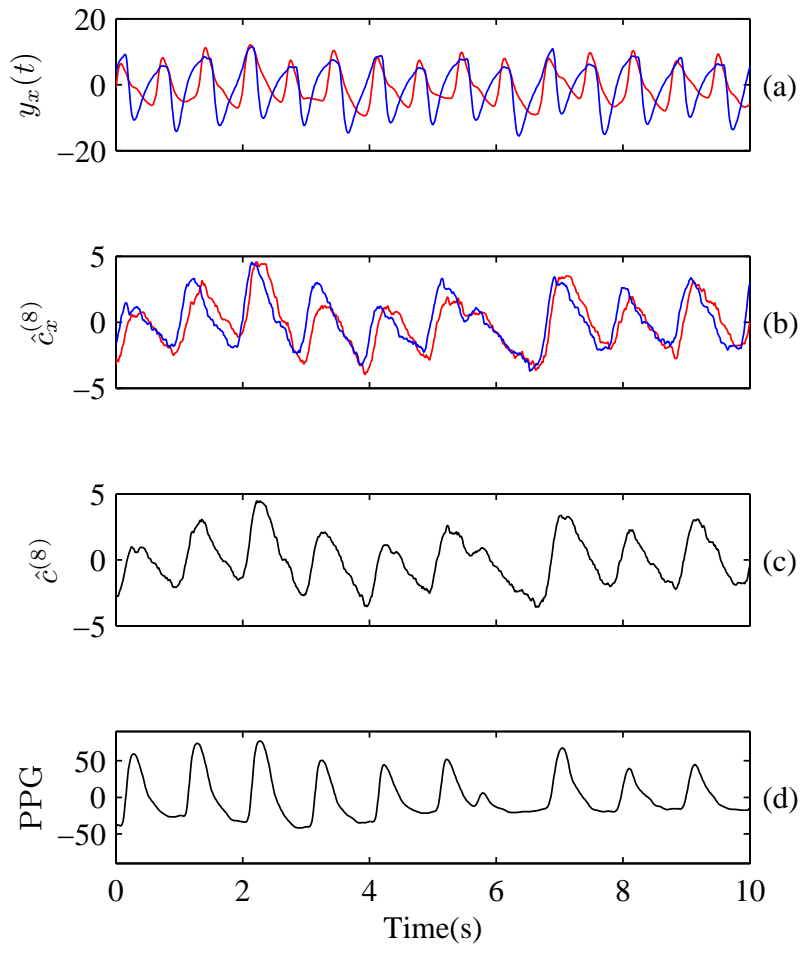

Figure 2. The measured arterial (red) and venous (blue) pressure signals are shown in (a), the arterial (red) and venous (blue) cardiac estimates in (b), the common cardiac estimate in (c), and the PPG signal in (d). Note that the vertical scales in (a)-(c) have $\mathrm{mmHg}$ as unit and (d) has arbitrary unit.

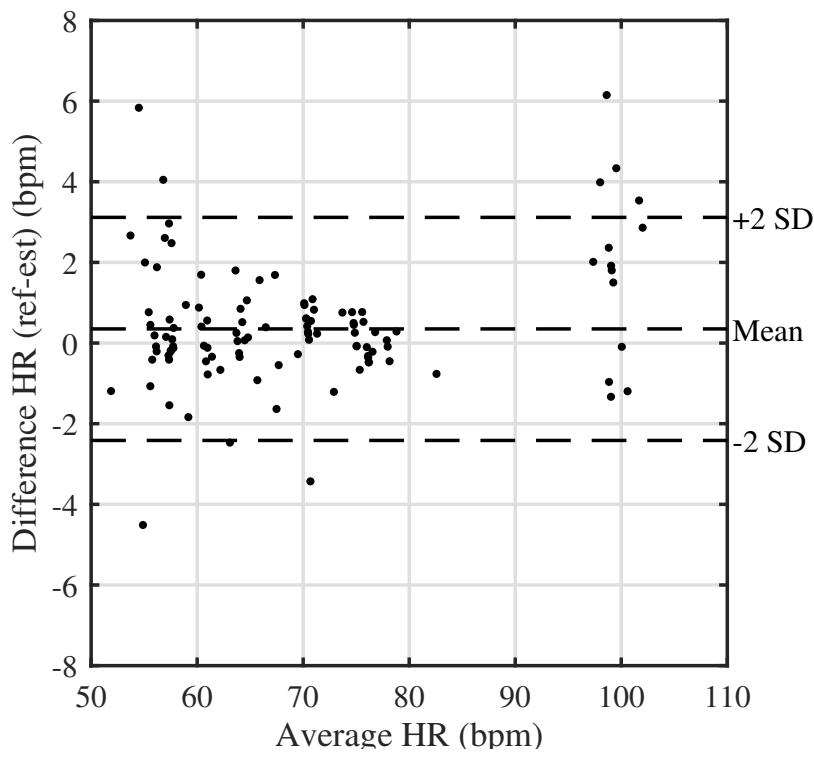

Figure 3. Bland-Altman plot of the difference between the heart rate extracted from both the arterial and venous pressure and the reference. 


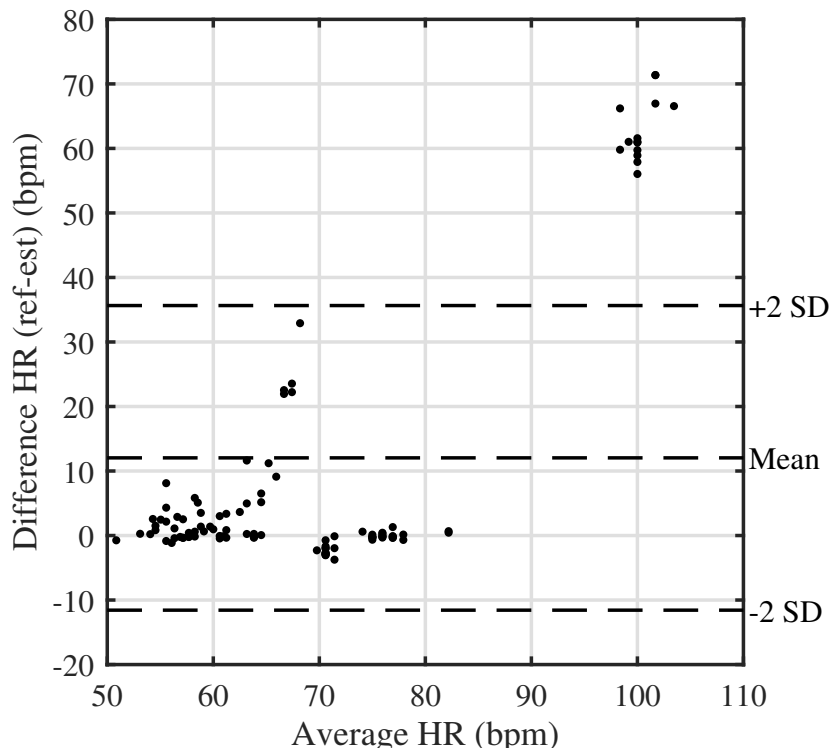

Figure 4. Bland-Altman plot of the difference between the heart rate extracted from the venous pressure and the heart rate determined from the reference.

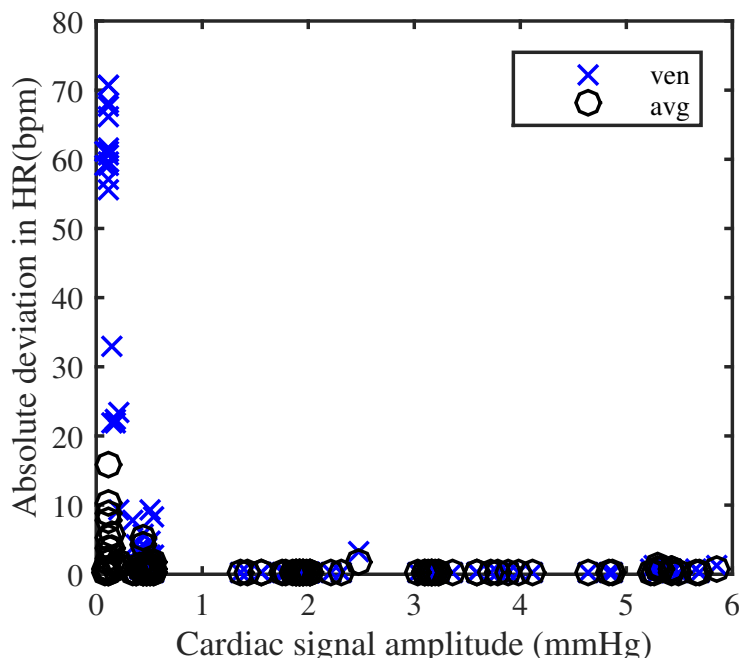

Figure 5. The absolute error in the estimates of the heart rate versus the cardiac signal amplitude. For each 1-min section, the errors obtained with the method using the venous signal (ven) are marked with blue checks, and the errors obtained with the new method using both arterial and venous signals (avg) are marked with black circles.
Hospital, Sweden, and Per Hansson, Baxter International Inc., for carrying out the clinical investigation.

\section{References}

[1] US Renal Data System. Patient mortality and survival. Am J Kidney Dis 1996;28:79-92.

[2] Selby NM, McIntyre CW. The acute cardiac effects of dialysis. Semin Dial 2007;20:220-228.

[3] Sörnmo L, Sandberg F, Gil E, Solem K. Noninvasive techniques for prevention of intradialytic hypotension. IEEE Rev Biomed Eng 2012;5:45-59.

[4] Moissl U, Wabel P, Leonhardt S, Isermann R, Krämer M. Continuous observation and analysis of heart rate during hemodialysis treatment. In Proc. Eur. Med. \& Biol. Eng. Conf. (EMBEC), volume 37. 1999; S558-S559.

[5] Moissl U, Wabel P, Leonhardt S, Isermann R, Krämer M. Heart rate monitoring during hemodialysis treatment. In Proc. Eur. Med. \& Biol. Eng. Conf. (EMBEC), volume 3. 2002; 584-585.

[6] Holmer M, Sandberg F, Solem K, Grigonyte E, Olde B, Sörnmo L. Extracting a cardiac signal from the extracorporeal pressure sensors of a hemodialysis machine. IEEE Trans Biomed Eng 2015;62(5):1305-1315.

[7] Lázaro J, Gil E, Vergara JM, Laguna P. Pulse rate variability analysis for discrimination of sleep-apnea-related decreases in the amplitude fluctuations of pulse photoplethysmographic signal in children. IEEE J Biomed Health Inform 2014;18:240-246.

Address for correspondence:

Mattias Holmer

Dept. of Biomedical Engineering, Lund University, Box 118, SE-221 00 Lund, SWEDEN mattias.holmer@bme.lth.se 